\title{
Application of Terminalia catappa in Preparation of Silver Nanoparticles to Develop Antibacterial Nylon
}

\author{
ELI ROHAETI ${ }^{* *}$ and ANNA RAKHMAWATI ${ }^{2}$ \\ 'Department of Chemistry Education, Faculty of Mathematics and Natural Science, Yogyakarta \\ State of University, 55281 Indonesia. \\ ${ }^{2}$ Department of Biology Education, Faculty of Mathematics and Natural Science, Yogyakarta \\ State of University, Indonesia. \\ *Corresponding author E-mail: eli_rohaeti@uny.ac.id \\ http://dx.doi.org/10.13005/ojc/330625
}

(Received: September 09, 2017; Accepted: October 15, 2017)

\begin{abstract}
Preparation of silver nanoparticle by using leaf extracts of Terminalia catappa, its deposition on Nylon fabrics, and the modification with an addition of HDTMS have been conducted in this research. The samples prepared in this study were nylon cloth (N0), nylon cloth - silver nanoparticles (N1), Nylon cloth - HDTMS (N2), Nylon cloth - silver nanoparticles - HDTMS (N3), and Nylon cloth - HDTMS - silver nanoparticles (N4). The silver nanoparticles were performed by using UvVis spectrophotometer, antibacterial activity of Nylon against S.aureus and E.coli was determined by measuring a diameter of clear zone, and water contact angle of the sample was measured by a sessile drop method. Silver nanoparticles are successfully produced using Terminalia catappa extracts as indicated by the absorption peaks at $448.50 \mathrm{~nm}$. Samples of Nylon cloth-silver nanoparticles - HDTMS (N3) show the highest antibacterial activity against $S$. aureus and $E$. coli with a strength 3 to 13 times greater than silver nanoparticles. Each sample of N1, N2, N3, and N4 shows the same ability to inhibit the growth of S.aureus and E.coli. The addition of HDTMS increases contact angle of nylon cloth.
\end{abstract}

Keywords: Antibacterial activity, Nylon, Self cleaning textile, Silane, Silver nanoparticles.

\section{INTRODUCTION}

The self-cleaning textiles with antibacterial properties are very useful for the medical world especially those used by patients, doctors, and nurses in a hospital. The use of polyamide fibers in the field of apparel textiles is wide enough such as socks, underwears, sportswear, to the use of techniques such as tire reinforcement yarn, tarpaulins, towing belts and as a denture base material ${ }^{1}$. Polyamide or Nylon fabric is one example of the first synthetic polymer made by Wallace Carothers, produced from hexamethylenediamine and adipic acid. On the reaction, it is produced a fiber called "Nylon 6.6".

Development of the self-cleaning and the antibacterial textile materials needs to be done to have a better quality of the products. The product of self-cleaning textile can be obtained by imitating 
Lotus leaf surface (Nelumbo nucifera) that has a complex surface texture between micro to nano scale or hydrophobic. This causes the dirt on the surface of the textile will be easily separated without making the textile to be wet ${ }^{2}$. Textile materials having hydrophobic properties can be obtained by modifying those with a silane compound. Silanebased molecules have long hydrocarbon chains and have a low enough surface energy to make a hydrophobic textile material ${ }^{3}$.

The antibacterial properties of textile materials can be obtained by utilizing nanotechnology, by depositing nanometer-sized particles onto a textile material. The development is currently quite rapid, and it is expected that the application of nanotechnology can improve the competitiveness of the national textile industry ${ }^{4}$. The application of nanotechnology or the use of nanometer-scale chemicals for textile materials in the process of refinement and improvement will produce a more functional textile material than conventional textiles ${ }^{2}$. To make antibacterial or antimicrobial textile materials necessary on nanoparticles are appropriate, the nanoparticles used should be able to kill various types of microbes in a broad spectrum, but not toxic to non-pathogenic microbes. In some literatures, it has been disclosed that silver nanoparticles coating on textile materials such as polymer fibers i.e cotton and Nylon fibers can make the textile material becoming antibacterial ${ }^{4}$.

Silver nanoparticles can be obtained by several methods, such as chemical reduction, electrochemistry, photochemistry, and sonochemistry. The most common method used is the chemical reduction. The several factors that make the method popular are because of the convenience factor, the relatively cheap cost and it is possible to produce on a large scale. The silver nanoparticles which were prepared by using polyvinylpyrrolidone (PVP) are spherical and relatively uniform ${ }^{5}$. The chemical reduction method for obtaining silver nanoparticles was performed by using silver salts and sodium citrate or sodium borohydrate ${ }^{6}$.

There is now another method of preparation of nanoparticle by utilizing living things such as microorganisms and plants as the reductors. The synthesis of these nanoparticles is known as the green synthesis of nanoparticles ${ }^{7}$. The method which uses the principle of green chemistry, is growing and widely used today because the chemical reduction process is feared to produce highly toxic properties when applied in the field of biomedicine. The purpose of the method is to replace toxic substances in chemical reduction processes ${ }^{8}$.

In this research, a fabric or textile material which is a polymer fiber was developed into selfcleaning and antibacterial textile product. The product of self-cleaning textile can be produced by the addition of silane compound to be hydrophobic. Meanwhile, the antibacterial textiles can be produced by the addition of nanoparticles. Silver nanoparticles were obtained by biosynthetic methods using plant extracts of Ketapang (Terminalia catappa) leaves ${ }^{9}$. In this method of reduction, the extract of Ketapang leaves acts as a bioreductor.

\section{MATERIALS AND METHODS}

\section{Materials and Procedures}

Nylon fabrics were purchased from the fabric store in Yogyakarta, Indonesia. Silver nitrate, polyvinyl alcohol (PVA), ethanol, acetone, and hexadecyltrimethoxysilane (HDTMS) were purchased as commercial products and used as they were without any further purification. Nutrient Agar (NA) and Nutrient Broth (NB) were purchased from Oxoid. Staphylococcus aureus ATCC 25923 and Escherichia coli 32518 were obtained from a collection of Faculty of Medicine, Gadjah Mada University.

The study was conducted in the following stages: extraction of Ketapang Leaf (Terminalia catappa), preparation of silver nanoparticle, deposition of silver nanoparticle on Nylon fabrics, modification of Nylon fabrics with the addition of HDTMS, and characterization of Nylon fabrics.

\section{Preparation of silver nanoparticle by using Terminalia catappa}

About $20 \mathrm{~g}$ of Terminalia catappa leaves were washed with aquadest, put into a $500 \mathrm{ml}$ beaker glass then $100 \mathrm{ml}$ of distilled water was added to extract on boiling for about 20 minutes. It was then filtered using Whatman No. 42 at room temperature. To about $1 \mathrm{ml}$ of the extract, $40 \mathrm{ml}$ of silver nitrate solution $1.10^{-3} \mathrm{M}$ was added. The mixture was left 
for about $2 \mathrm{~h}$. to react and then about $12 \mathrm{ml}$ of $1 \%$ of PVA solution was added while stirring for about 2 hours. The solution was then allowed to stand for 4 days to produce a colloidal silver nanoparticle. The colloidal was characterized using a UV-Vis spectrophotometer.

\section{Application of silver nanoparticles on Nylon fiber (Nylon - Ag)}

Nylon fabric was cut to the size of $5 \mathrm{~cm} \mathrm{x}$ $5 \mathrm{~cm}$. It was washed by soaking in acetone for $30 \mathrm{~min}$. and then rinsed with or soaked in distilled water nonion for about $30 \mathrm{~min}$. and dried using a hairdryer. Then, Nylon fiber was immersed in the colloidal of silver nanoparticle, twisted around using a shaker with a speed of $153 \mathrm{rpm}$ for $24 \mathrm{~h}$ and finally dried at room temperature.

\section{Modification of Nylon fiber surface with HDTMS (Nylon - HDTMS)}

HDTMS was dissolved in ethanol. Then, Nylon and the Nylon-Ag were immersed into the $4 \%$ of HDTMS solution. The mixture was allowed to react for about $6 \mathrm{~h}$ at room temperature. Nylon and Nylon-Ag immersed in silane solution were twisted using shaker at $155 \mathrm{rpm}$ for $1 \mathrm{~h}$ followed by drying at room temperature. Then, Nylon fabric before and after modification was analyzed by using antibacterial activity test and contact angle test. The sample which was prepared in this study were labeled as the followings, Nylon cloth (N0), Nylon cloth - silver nanoparticles (N1), Nylon cloth - HDTMS (N2), Nylon cloth - silver nanoparticles - HDTMS (N3), and Nylon cloth - HDTMS - silver nanoparticles (N4).

\section{Test of antibacterial activity}

Antibacterial activity was performed by preparing bacterial growth in media such as Nutrient Agar and Nutrient Broth by dissolving $14 \mathrm{~g}$ of NA in $500 \mathrm{ml}$ of distilled water and $1.3 \mathrm{~g}$ of NB in $250 \mathrm{ml}$ of distilled water. All the tools and media for growing bacteria were sterilized in autoclave. Rejuvenation of Staphylococcus aureus ATCC25923 and Escherichia coli ATCC32518 were performed on an agar medium NA and incubated for $24 \mathrm{~h}$ at room temperature. Staphylococcus aureus 25923 and Escherichia coli ATCC32518 which has been rejuvenated for 24 Escherichia coli and Staphylococcus aureus were analyzed by statistic test using SPSS version 15 program. The test was done by ANOVA test, LSD test, and t-Independent. ANOVA test was used to determine the effect of sample type, incubation time, and interaction between samples and incubation time on the antibacterial activity. LSD test was used to determine the significance between samples, and a t-Independent test was used to determine whether there were significant differences in antibacterial activity of Nylon samples between Escherichia coli (Gram negative) and Staphylococcus aureus (Gram positive).

\section{Test of water contact angle}

The hydrophobic properties of the samples were determined by measuring the water contact angle $(\theta)$ between the fluid and the sample surface using sessile drop method ${ }^{10}$. Samples were placed on the surface of a table or a flat board and micropipette was placed on the top then paired with the upright. By using a pipette, a water was dripped from a height of $1 \mathrm{~cm}$ of the sample. Once the water dripped, the contact angle shooting was done using the camera with adjustable contrast, light, and focus settings. The images were processed using Corel Draw software $\mathrm{X} 4$ version to determine the contact angle automatically between the liquid surface and the sample.

\section{RESULTS AND DISCUSSION}

\section{Characteristic of Silver Nanoparticles}

The extracts of Terminalia catappa leaves and silver colloidal of silver nanoparticles in this synthesis are shown in Fig.1. The leaves extract after the addition of silver nitrate solution and PVA solution changed from colorless to dark brown. The color change of the solution occurring due to the $-\mathrm{OH}$ group in the tannin as the main component of Terminalia catappa extract has oxidized to the $\mathrm{C}=\mathrm{O}$ group. It indicates that reduction process of silver nitrate solution has occurred and formed colloidal of silver nanoparticles.

The analysis using UV-Vis spectrophotometer in this study was conducted at the wavelength range 190-600nm for an $\mathrm{AgNO}_{3}$ solution and 200-600nm for the analysis of colloidal silver nanoparticles. The UV-Vis spectra patterns are presented in Fig.2. The $\mathrm{AgNO}_{3}$ solution shows the absorption band 


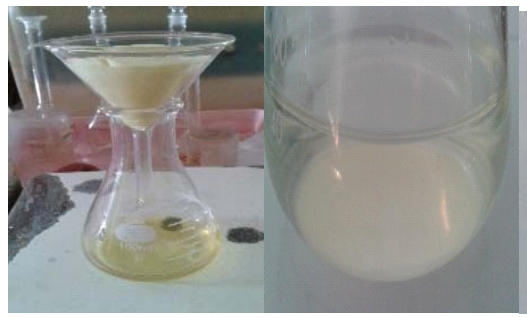

(a)

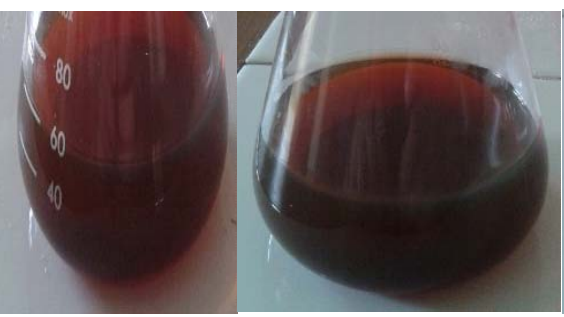

(c)

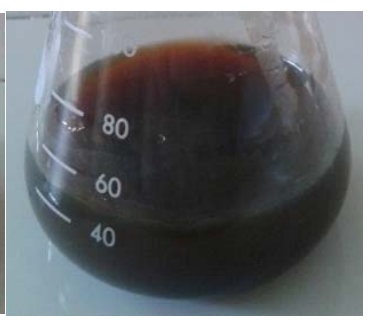

(e)

Fig.1. The extracts of Terminalia catappa leaves (a), extracts of leaves $+\mathrm{AgNO}_{3}$ solution + PVA solution (b), silver colloidal after 1,3 , and 4 days (c, d, and e)

peak at a wavelength of $218.50 \mathrm{~nm}$. While the silver nanoparticles show three band peaks. The first band peak that appears at a wavelength of $214 \mathrm{~nm}$ indicates that it's still $\mathrm{Ag}^{+}$which could not be reduced by leaf extract to $\mathrm{Ag}^{0}$. While the second band peak that appears at $254 \mathrm{~nm}$ and the third

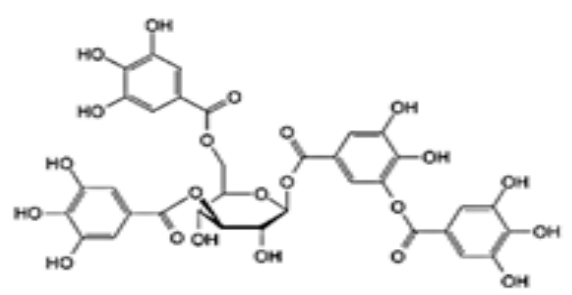

(a) band peak at $448.50 \mathrm{~nm}$ show $\mathrm{Ag}^{+}$has been successfully reduced to $\mathrm{Ag}^{0}$ by leaf extract. The leaf extract of Terminalia catappa has tannins compounds, that are thought to play an important role as the reducing agents, the reaction mechanism is estimated as in Figure. 3.

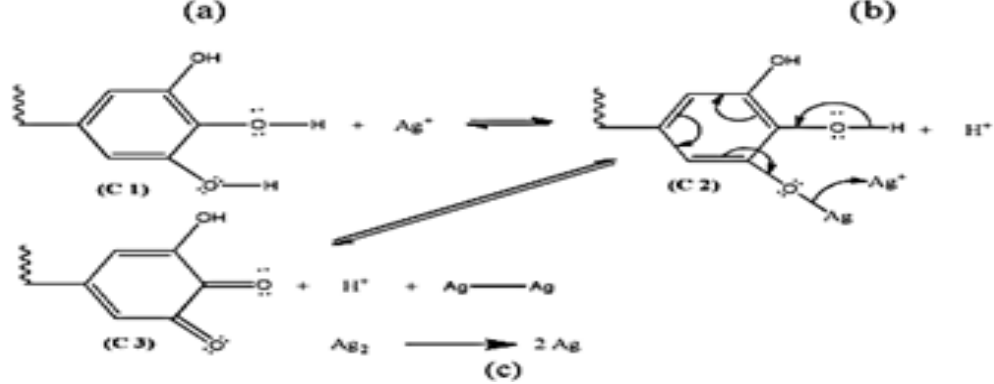

Fig.3. Molecular structure of Tannins (a), simple structures of Tannins (b), and reaction mechanisms of AgNP establishment (c) $)^{11}$

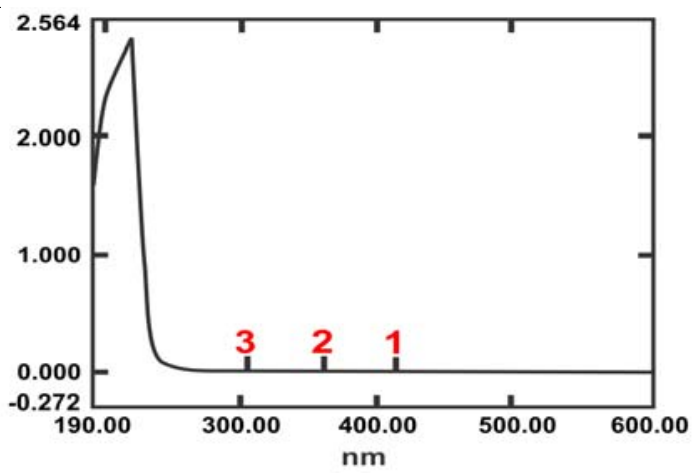

(a)

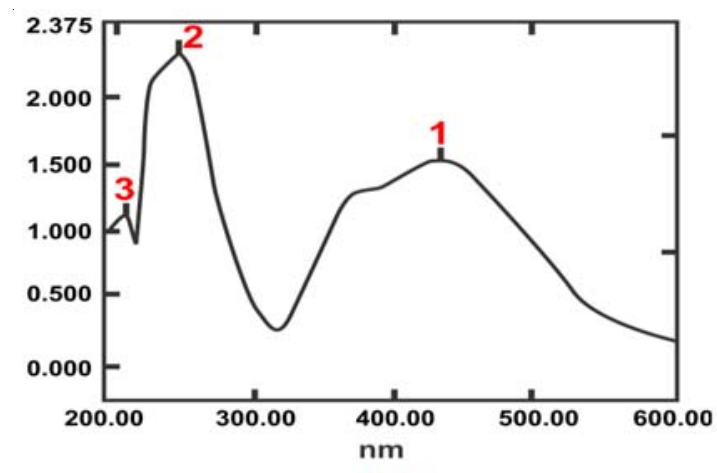

(b)

Fig.2. The Uv-Vis spectra of $\mathrm{AgNO}_{3} 1.10^{-3} \mathrm{M}(\mathrm{a})$ and silver nanoparticles (b) 
Antibacterial activity of Nylon against Staphylococcus aureus ATCC 25923 and Escherichia coli ATCC 35218

Figure. 4 shows the diameter of the clearzone formed of Nylon 6.6 fabrics against Staphylococcus aureus ATCC 25923 and Escherichia coli ATCC 35218. The clear zone formed shows that bacteria do not grow in the area. Clear zone measurements were performed for $72 \mathrm{~h}$ of incubation. Data from observation of clear zone on samples N0, N1, N2, $\mathrm{N} 3$, and N4 in inhibiting the growth of Staphylococcus aureus ATCC 25923 are presented in Table. 1.

Table. 1: Diameter of clear zone of Nylon against Staphylococcus aureus ATCC 25923

\begin{tabular}{lccccc}
\hline $\begin{array}{l}\text { Time } \\
\text { (hours) }\end{array}$ & \multicolumn{5}{l}{$\begin{array}{l}\text { Diameter of Clear Zone }(\mathrm{mm}) \\
\text { against Staphylococcus aureus } \\
\text { ATCC 25923 }\end{array}$} \\
\hline \multicolumn{7}{c}{ N0 } & N1 & N2 & N3 & N4 \\
\hline 42 & 11.2 & 22.2 & 19.2 & 25.3 & 12.8 \\
48 & 13.1 & 24.4 & 22.3 & 30.5 & 21.5 \\
54 & 14.7 & 24.1 & 23.4 & 28.2 & 17.7 \\
60 & 14.0 & 23.7 & 18.9 & 12.5 & 16.1 \\
66 & 13.8 & 21.7 & 17.8 & 10.7 & 15.6 \\
72 & 17.2 & 16.7 & 14.5 & 6.70 & 12.3 \\
\hline
\end{tabular}

The sample of $\mathrm{N} 3$ shows the highest antibacterial activity compared to other cloth samples at $48 \mathrm{~h}$ of incubation. This suggests that the addition of silver nanoparticles and HDTMS increases the antibacterial activity of Nylon fabrics to inhibit the growth of Staphylococcus aureus. The sample of N3 has much higher antibacterial activity than silver nanoparticles. Whereas silver nanoparticles are a good antibacterial ingredient ${ }^{12}$. Silver nanoparticles demonstrated a diameter of the clear zone as much as $2.3 \mathrm{~mm}^{12}$. Thus, the samples of N3 at $48 \mathrm{~h}$ of incubation can be used as antibacterial agents in inhibiting $S$. aureus with a strength 13 times greater than silver nanoparticles. The diameter of a clear zone of the N3 sample at $72 \mathrm{~h}$ incubation still showed antibacterial activity 3 times greater than the antibacterial activity of the silver nanoparticles.

Table. 2 shows clear zone on samples N0, N1, N2, N3, and N4 in inhibiting the growth of Escherichia coli ATCC 35218. The sample of N3 shows the highest antibacterial activity compared to other cloth samples at $60 \mathrm{~h}$ of incubation. This suggests that the addition of silver nanoparticles and HDTMS increases the antibacterial activity of Nylon fabrics to inhibit the growth Escherichia coli. The sample of N3 has much higher antibacterial activity than silver nanoparticles. A diameter of the clear zone of silver nanoparticles was as much as $2.3 \mathrm{~mm}$ against $E$. colit2. Thus, the samples of $\mathrm{N} 3$ at $60 \mathrm{~h}$ of incubation can be used as antibacterial agents in inhibiting $E$. coli with a strength 13.5 times greater than silver nanoparticles.

Table. 2: Diameter of clear zone of Nylon against Escherichia coli ATCC 35218

\begin{tabular}{lccccc}
\hline $\begin{array}{l}\text { Time } \\
\text { (hours) }\end{array}$ & \multicolumn{5}{c}{$\begin{array}{l}\text { Diameter of Clear Zone (mm) against } \\
\text { Escherichia coli ATCC } 35218\end{array}$} \\
\hline & N0 & N1 & N2 & N3 & N4 \\
\hline 42 & 15.1 & 10.7 & 13.1 & 15.4 & 10.3 \\
48 & 19.1 & 18.4 & 24.9 & 26.9 & 22.3 \\
54 & 18.0 & 20.5 & 23.5 & 25.6 & 21.1 \\
60 & 20.1 & 23.1 & 25.3 & 31.1 & 21.1 \\
66 & 21.9 & 22.7 & 23.4 & 28.7 & 21.3 \\
72 & 17.6 & 17.9 & 17.5 & 14.5 & 14.9 \\
\hline
\end{tabular}

The N3 has the highest antibacterial activity in inhibiting the growth of $S$. aureus and $E$. coli. The silver nanoparticles inhibit and destroy a microorganism through a mechanism, beginning with silver nanoparticles that release $\mathrm{Ag}^{+}$, then the $\mathrm{Ag}^{+}$will interact with the thiol group (-SH) on the surface protein, followed by the replacement of the hydrogen cation $\left(\mathrm{H}^{+}\right)$of the thiol group to give a more stable $\mathrm{S}-\mathrm{Ag}$ group. This will disable the protein, and decrease the membrane permeability. The next process is that silver ions will enter the cell and change the DNA structure and ultimately lead to cell death ${ }^{13}$

The formation of high-energy molecules such as ATP is essential for bacterial metabolism. In the ATP molecule, there is a high-potency of group transfer that is a phosphate group, the phosphate group is easily transferred when attacked by more negative molecules (nucleophiles) as hydroxyl groups ${ }^{14}$. HDTMS compounds have hydroxyl groups so that the hydroxyl group can attack or react with the phosphate group and disrupt the process of energy formation in bacteria, causing the growth of bacteria to be inhibited. The effect of HDTMS compounds in inhibiting the bacterial growth may 
also be due to the nature of HDTMS compounds being similar to detergents that are hydrophilic and hydrophobic. In addition, HDTMS compounds can also reduce the surface tension as well as detergents. The detergent containing hydrophilic and hydrophobic groups are antibacterial agents that can damage cytoplasmic membranes and kill bacterial cells ${ }^{15}$.

The result of ANOVA test is presented in Table.3., the result of LSD test is presented in Table.4., and the result of the t-independent test is presented in Table.5. The incubation time affects the antibacterial activity of the Nylon sample in inhibiting the growth of $S$. aureus and E. coli (Table. 3 and Table.4). The type of fabric affected antibacterial activity only in inhibiting the growth of S. aureus. Each type of cloth and incubation time affects the antibacterial activity of the fabric in inhibiting $S$. aureus bacteria. Thus, the method of modification of the Nylon fabric through the addition of silver nanoparticles and HDTMS compounds affects the antibacterial activity properties of the modified fabric significantly. However, the time of incubation and the type of Nylon cloth did not simultaneously affect the antibacterial activity of the cloth in inhibiting the growth of each bacterium.

Table. 3 :The effect of time and type of sample to antibacterial activity of Nylon against Staphylococcus aureus and Escherichia coli

\begin{tabular}{lccccc}
\hline \multicolumn{6}{c}{ Staphylococcus aureus ATCC 25923} \\
Source of data & Sum & Df & Average & F & Sig. \\
\hline Time & 0.086 & 5 & 0.017 & 3.449 & 0.008 \\
Sample & 0.072 & 4 & 0.018 & 3.616 & 0.010 \\
Time and Sample & 0.120 & 20 & 0.006 & 1.197 & 0.289 \\
\hline
\end{tabular}

Escherichia coli ATCC 35218

\begin{tabular}{lccccc}
\hline Source of data & Sum & Df & Average & F & Sig. \\
\hline Time & 0.151 & 5 & 0.030 & 5.403 & 0.000 \\
Sample & 0.037 & 4 & 0.009 & 1,647 & 0.174 \\
Time and Sample & 0.033 & 20 & 0.002 & 0.294 & 0.998 \\
\hline
\end{tabular}

As in Table. 4, sample N3 and N4 show a significant difference in inhibiting the growth $E$. coli.

Table. 4: Interpretation of the result of LSD test between samples type against Staphylococcus aureus and Escherichia coli

\begin{tabular}{lcc}
\hline Type of & \multicolumn{2}{c}{ Conclusion } \\
sample & E. coli & S. aureus \\
\hline N0 - N1 & Not significant & Significant \\
N0 - N2 & Not significant & Significant \\
N0 - N3 & Not significant & Significant \\
N0 - N4 & Not significant & Not significant \\
N1 - N2 & Not significant & Not significant \\
N1 - N3 & Not significant & Not significant \\
N1 - N4 & Not significant & Significant \\
N2 - N3 & Not significant & Not significant \\
N2 - N4 & Not significant & Not significant \\
N3 - N4 & Significant & Not significant \\
\hline
\end{tabular}

This means modification of Nylon fabric by using 2 types of materials affecting the antibacterial activity of the fabric. Coating the silver nanoparticles first, and then coating with the HDTMS compound show better fabric antibacterial activity in inhibiting the growth of $E$. coli (Table. 2). While between sample $\mathrm{N} 0$ - N1, N0 - N2, N0 - N3, and N1-N4 show a

Table. 5: Interpretation of the result of t-Indepndent against Staphylococcus aureus ATCC 25923 and Escherichia coli ATCC 35218

\begin{tabular}{lc}
\hline Nylon & t-Independent \\
\hline N0 & Difference \\
N1 & No difference \\
N2 & No difference \\
N3 & No difference \\
N4 & No difference \\
\hline
\end{tabular}


significant difference in inhibiting the growth $S$. aureus. This shows that modification of Nylon fabric by adding the silver nanoparticles, HDTMS compound, and also the silver nanoparticle and HDTMS compound can increase antibacterial activity of the Nylon fabrics (N0) significantly against S. aureus. Between Nylon - Ag (N1) and Nylon -HDTMS - Ag (N4) have the different antibacterial activity significantly. $\mathrm{N} 1$ has the higher antibacterial activity than N4 against $S$. aureus (Table.1). It means the addition of HDTMS into fabrics first in modification Nylon fabrics can decrease their antibacterial activity. In Table.5, sample N1, N2, N3, and N4 show the same ability to inhibit the growth of both $S$. aureus and $E$. coli, while NO shows significantly different in inhibiting the growth between the two, S. aureus and E. coli.

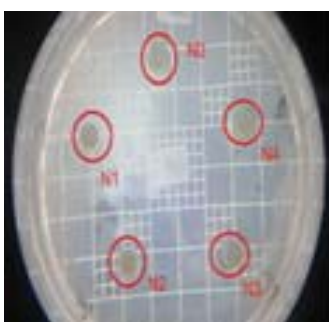

(a)

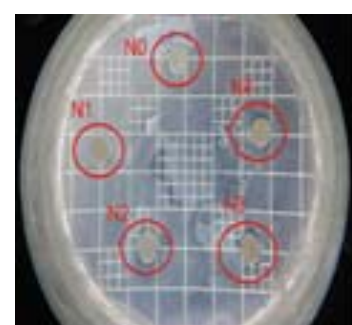

(b)
Fig.4. Diameter of clear zone of Nylon against Staphylococcus aureus ATCC 25923 (a) and Escherichia coli ATCC 35218 (b)

The water contact angle of Nylon

The water contact angle of Nylon before and after modification with the addition of silver nanoparticles and HDTMS compound are presented in Fig. 5. Sample N2 shows the highest contact angle $\left(120.75^{\circ}\right)$ in this study. Therefore, the addition of HDTMS compound clearly can increase the contact angle of Nylon.

The HDTMS compound is an amphiphilic molecule with a hydrophilic head section, $\left(\mathrm{Si}\left(\mathrm{OCH}_{3}\right)_{3}\right)$, and a tail portion which is a hydrophobic long alkyl group $\left(\mathrm{C}_{16} \mathrm{H}_{33}\right)$. This compound can provide a low surface free energy ${ }^{16}$. The HDTMS compound that was superimposed on Nylon fabric sample will interact and form a bond that results in free surface energy down so that the surface of the fabric sample will be hydrophobic. Initially, the HDTMS compound superimposed on a material surface that will be hydrolyzed and produce the $-\mathrm{OH}$ group. The hydrolysis reaction of HDTMS is as follows ${ }^{16}$ :

$\mathrm{C}_{16} \mathrm{H}_{33} \mathrm{Si}\left(\mathrm{OCH}_{3}\right)_{3}+\mathrm{H}_{2} \mathrm{O} \rightarrow \mathrm{C}_{16} \mathrm{H}_{33} \mathrm{Si}(\mathrm{OH})_{3}+3 \mathrm{CH}_{3} \mathrm{OH}$
The $-\mathrm{OH}$ group of HDTMS will form a bond with a typical group of Nylon fabric surfaces i.e -CONH- forming Si-O-N bonds. As a result of the bonding, the tail of the HDTMS is a long hydrophobic alkyl group that extends outward, and becomes a barrier for water molecules soaking the fabric surface so that the Nylon fabric will be hydrophobic.

The addition of silver nanoparticles to a Nylon fabric also coated with HDTMS causes a decrease in contact angle. The decrease of the contact angle value due to the silver nanoparticles on the sample surface can be resulted from the contact of the HDTMS on the surface of the Nylon fabric being smaller. The silver nanoparticles deposited on the surface of the Nylon fabric have a nanometer size and the smaller the size of a material the smaller the contact angle would be. The surface area will be even greater so that HDTMS compounds can't coat the surface of the fabrics perfectly.

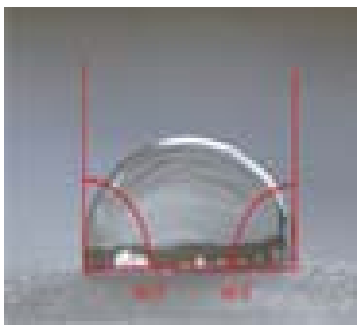
$\theta=90.00$

(a)

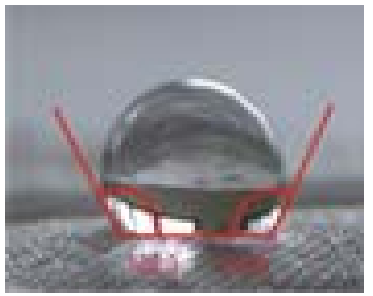

$\theta=120.75$

(c)

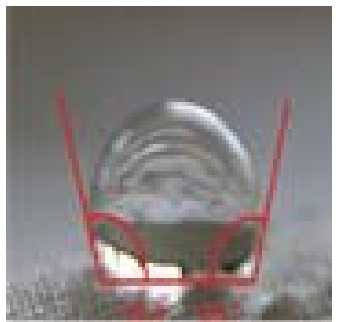

$\boldsymbol{\theta}=107.30$

(b)

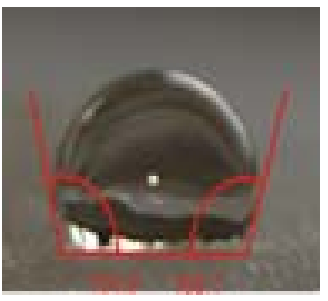

$\theta=106.50$

(d)

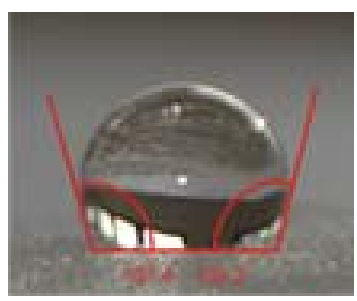

$\theta=106.35$

(e)

Fig.5. The water contact angle of NO (a), N1 (b), N2 (c), N3 (d), and N4 (e) 


\section{CONCLUSIONS}

Silver nanoparticles were successfully produced using Terminalia catappa extracts as indicated by the absorption band peak at $448.50 \mathrm{~nm}$.

Samples of Nylon cloth - silver nanoparticles - HDTMS showed the highest antibacterial activity against $S$. aureus and $E$. coli with a strength 3 to 13 times greater than silver nanoparticles.

The incubation time affected the antibacterial activity of the Nylon sample in inhibiting the growth of $S$. aureus and E. coli. The time of incubation and the type of Nylon cloth did not simultaneously affect the antibacterial activity of the cloth in inhibiting the growth of each bacterium.
Each sample of N1, N2, N3, and N4 showed the same ability to inhibit the growth of both $S$. aureus and E. coli, while NO showed significantly different to inhibit the growth between the two, $S$. aureus and E. coli.

Nylon cloth - HDTMS (N2) showed the highest contact angle.

\section{ACKNOWLEDGEMENTS}

The authors thank the finance support from Ministry of Research, Technology and Higher Education of the Republic Indonesia through project Fundamental Research 2017.

\section{REFERENCES}

1. Vojdani, M. and Giti, R. J. Dent. 2015, 16(1), 1-9

2. Wahyudi, T and Rismayani, S. Arena Tekstil, 2008, 23(2), 84-90

3. Khalilabad, M.S. and Yazdanshenas, M. E. Cellulose. 2013, 20, 3039-3051

4. Haryono, A and Harmami, S. R. Jurnal Kimia Indonesia. 2010, 5(1), 1-6.

5. Alahmad, A.; Eleoui, M.; Falah, A.; Alghoraibi, I. Phys. Sci. Res. Int. 2013, 1(4), 89-96

6. Guzman, M.; Dille, J; Godet, S. Nanomed. 2012. 8(1), 37-45

7. Keat, C. L.; Aziz, A.; Eid, A. M.; Elmarzugi, N. A. Biores. and Bioproc. 2015, 2(47), https:// doi.org/10.1186/s40643-015-0076-2

8. Duran, N.; Marcato, P. D.; Souza, G. I. H. D.; Alves, O. L.; Esposito, E. J. Biomed. Nanotech. 2007, 3(2), 203-208

9. Lembang, E. Y.; Maming;; Zakir, M. Repository Jurnal Kimia FMIPA Universitas Hasanuddin, Indonesia. 2013. http://repository.unhas.ac.id/ handle/123456789/9268.
10. Morones, J. R.; Elechiguerra, J. L.; Camacho, A.; Holt, K.; Kouri, J. B.; Ramirez, J. T.; Yacaman, M. J. Nanotech. 2005, 16(1), 23462353

11. Zakir, M.; Maming, Lembang, E.; Lembang, M. Int. Conf. on Adv. Mat. and Prac. Nanotech. 2014, Jakarta, Indonesia.

12. Nair, A. S.; Binoy, N. P.; Ramakrishna, S.; Kurup, T. R. R.; Chan, L. W.; Goh, C. H.; Islam, M. R.; Utschig, T.; Pradeep, T. ACS Appl. Mater. Interfaces. 2009, 1, 2413-2419

13. Feng, Q. L.; Wu, J.; Chen, G. Q.; Cui, F. Z.; Kim, F. N.; Kim, J. O. J. Biomed. Mat. Res. 2000, 52(4), 662-668.

14. Hunter, T. Philos. Trans. R. Soc. Lond. B. Biol. Sci. 2012, 367(1602), 2513-2516 doi: 10.1098/rstb.2012.0013

15. Rama, B. P.; Prajna, P. S.; Menezez, V.P.; Shetty, P. Adv. In Bioresearch. 2011, 2(2) , 52-62

16. Dhotel, A. Thesis. University of Nebraska, Lincoln., 2010. 